\title{
1 JADE family proteins regulate proteasome abundance and activity
}

2

3 Lena Kathrin Ebert ${ }^{1}$, Sebastian Bargfrede ${ }^{1}$, Katrin Bohl ${ }^{1}$, Roman-Ulrich Müller ${ }^{1,2}$, Thomas Benzing ${ }^{1,2}$,

4 Bernhard Schermer ${ }^{1,2, \#}$

$5{ }^{1}$ Department II of Internal Medicine and Center for Molecular Medicine Cologne, University of Cologne,

6 Faculty of Medicine and University Hospital Cologne, Cologne, Germany.

$7{ }^{2}$ CECAD, University of Cologne, Faculty of Medicine and University Hospital Cologne, Cologne,

8 Germany.

$9 \quad$ \#corresponding author

10

11 Email: bernhard.schermer@uk-koeln.de

12

13 Running title: Jade proteins control proteasomal activity

14 Key words: PHD finger, kidney, proteasome, ubiquitination, primary cilium 


\section{Abstract}

16 JADE family proteins (JADE1/2/3) have been implicated in diverse cellular functions and signaling pathways ranging from WNT signaling and cell cycle control to cell death and complex transcriptional regulation through histone acetyl-transferase complexes. JADE proteins show a high degree of sequence similarity and share two PHD zinc finger domains. JADE1 interacts with cilia-associated proteins and has been implicated in cilia-related genetic disorders with kidney phenotypes. However, the function of the widely expressed JADE proteins at the molecular level is still elusive. Here we show that JADE proteins regulate proteasome abundance and activity. Using kidney cells as a model, we demonstrate that loss of either JADE protein resulted in increased expression of almost all components of the $26 \mathrm{~S}$ proteasome. Regulation occurred at the post-translational level and was not the consequence of transcriptional activation. Consistent with a role for JADE proteins in regulating overall proteasomal abundance, proteasomal activity was elevated in Jade-deficient cells, while exogenous expression of JADE1/2/3 decreased the level of proteasome activity. Coimmunoprecipitation experiments confirmed the interaction of proteasomal subunits with Jade1 suggesting a direct role of JADE proteins in regulating turnover, stability and abundance of the 26 s proteasome. These data may now explain the plethora of cellular roles that have been attributed to JADE proteins. 


\section{Introduction}

The plant-homeodomain (PHD) zinc finger protein JADE1 (PHF17; Gene for Apoptosis and Differentiation in Epithelia 1) has been initially identified as interactor of the tumor suppressor PVHL in a yeast two-hybrid screen (1). Mutations in VHL cause the autosomal-dominant von-Hippel Lindau (VHL) disease, a hereditary tumor syndrome associated with hemangioblastomas, pheochromocytomas and clear-cell renal cell carcinoma (ccRCC). Aberrations of VHL are also found in the majority of sporadic ccRCCs (2-5). It has been shown that JADE1 is stabilized by PVHL and this stabilization is diminished if $V H L$ carries pathogenic mutations $(1,6-8)$. Moreover, JADE1 has been described as a single unit E3 ubiquitin ligase targeting beta-catenin for degradation and thereby inhibiting canonical WNT signaling (9). Both high levels of $\beta$-catenin and low levels of JADE1 are related to poor prognosis in RCC patients (6). This supports the current notion of JADE1 as a putative tumor suppressor in the kidney, related to pVHL. In addition, JADE1 forms a protein complex with members of the NPHP protein family, in particular NPHP4. Mutation in the NPHP genes result in Nephronophthisis, an autosomal-recessive cystic kidney disease leading to kidney failure in children and adolescents (10). Similar to pVHL, NPHP4 stabilizes JADE1 and enhances inhibition of canonical WNT signaling (11). Similarly, JADE1 has been functionally linked to polycystin-1 (PC1) (12). PC1 is encoded by the PKD1 gene, mutations of which cause autosomal-dominant polycystic kidney disease (ADPKD), the most frequent cystic kidney disease in adults. PC1, NPHP proteins and pVHL are localized to primary cilia and disorders caused by mutation of these proteins are considered ciliopathies (13). Interestingly, JADE1 has been shown to co-localize with NPHP proteins at the ciliary base (11). Besides the connection to ciliopathy proteins and WNT signaling, JADE1 has been described as a cofactor for two histone acetyltransferases (HATs), HBO1 and its homologue Tip60, thereby inducing histone $\mathrm{H} 4$ acetylation $(14,15)$. This implicates functions of JADE1 that affect transcriptional and epigenetic regulations, DNA metabolism, as well as cell cycle regulation $(14,16,17)$. Surprisingly, a gene trap mouse model targeting Jade1 did not develop any major phenotype (18). This led to the hypothesis that additional proteins could functionally overlap and compensate for the loss of JADE1. Obviously, the additional members of the Jade protein family, JADE2 (PHF15) and JADE3 (PHF16), are key candidates for this compensation (18). The JADE genes, encoding for the three individual JADE proteins, are located on three different chromosomes (chr. 4, chr. 5, chr. X in the human genome and chr. 3, chr. 11 and chr. $X$ in the mouse genome). As we will demonstrate in detail, all three JADE proteins share a high percentage of sequence similarity and conserved protein domains (19). Little is known about specific functions of JADE2 and JADE3, but a few studies provide some evidence for similar pathways and functions within the JADE family: Similar to JADE1, JADE2 was suggested to act as an E3-ubiquitin ligase that targets the histone H3K4 demethylase LSD1 (20-22) and JADE3 has been 
found to be induced by WNT/ $\beta$-catenin and to have oncogenic properties in colon cancer (23). Moreover, all three JADE proteins are part of the $\operatorname{HBO} 1$ complex $(14,17,24)$.

While JADE family members have been implicated with a variety of different pathways and processes, their molecular function has never been directly compared in the same tissue or cell type. Here, we analyzed common and individual functions of Jade1, Jade2, and Jade3 in differentiated murine renal tubular epithelial cells. Using CRISPR/Cas9-mediated genome editing, we generated monoclonal lossof-function cell lines with two independent strategies for each of the Jade genes, which resulted in two deficient cell lines per Jade gene. After demonstrating the lack of Jade expression and the absence of any counter-regulation on mRNA and protein level, we performed unbiased proteomic, transcriptomic and in-depth bioinformatic analyses to understand the individual alterations upon loss of Jade1, Jade2 and Jade3. Taken together, we identified and confirmed increased proteasomal abundance and activity as a common molecular phenotype of JADE-deficient cells.

\section{Results}

JADE protein family members share common interactors related to renal disease.

JADE proteins share two PHD zinc finger domains, several PEST domains, and nuclear localization signals (Fig. 1A). Based on their structural similarities, we sought to investigate whether JADE2 and JADE3 share molecular functions with JADE1. JADE1 has been characterized as an interactor of the ciliary protein pVHL (7) and of the nephrocystin protein complex (11). Therefore, we cloned the cDNA of human JADE2 and JADE3 in expression vectors and performed coimmunoprecipitation experiments, which included JADE1S as positive control. V5-tagged pVHL (V5.VHL) was co-expressed together with JADE1S (F.Jade1S), JADE2, JADE3, or a control protein, all with an N-terminal Flag-tagged. These experiments revealed pVHL to co-precipitate with all three JADE proteins (Fig. 1B). We performed similar experiments with V5.NPHP4 (Fig. 1C) and V5.NPHP1 (Fig. 1D). Again, both NPHP4 and NPHP1 specifically co-precipitated with all of the JADE proteins. Additional co-immunoprecipitation experiments with differently-tagged JADE proteins supported the idea, that JADE proteins form homodimers or homomultimers (Fig. 1E,F). Given the high structural similarity between the individual JADE proteins we assumed that JADE1/2/3 could also built hetero-multimeric protein complexes. This notion was confirmed by coimmunoprecipitation among the different JADE proteins (Fig. 1G). In summary, all JADE proteins share relevant renal protein complexes, very similar to their putative common role as co-factors and components of the HBO1/HAT complex $(14,24)$, indicating that they could interact in the same complex with a common function. (1) 99 
JADE family members show ubiquitous transcription with spikes in different tissues.

The N-terminal part of the JADE proteins shows the highest level of similarity as illustrated by the higher protein identity score between JADE2/3 and JADE1S compared to JADE1L (Supplemental Fig. S1A) (19). We used available data sets of the Human Protein Atlas to analyze tissue expression. Protein expression data was only available for JADE1 and shows global expression in most tissues with squamous epithelial cells of the oral mucosa, esophagus, and uterine, as well as hepatocytes and kidney tubules expressing the highest levels (Supplemental Fig. S1B). mRNA expression data support the ubiquitous expression of JADE1 in most tissues with higher levels in the pancreas and endocrine and female tissues (Fig. S1C). JADE2 appears ubiquitously expressed in most tissues with enrichment in the brain, endocrine tissues, female tissues, and lymphoid tissue. The overall JADE3 expression is relatively low as compared to JADE1 and JADE2. The expression pattern is similar to JADE2 with the highest levels in the brain, endocrine tissues, female and male tissues, and lymphoid tissues (Supplemental Fig. S1C). Importantly, while the expression of all three JADE genes is confirmed in most tissues, there is generally one JADE family member predominantly expressed (e.g., JADE1 in pancreas, bone marrow, or breast; JADE2 in cerebral cortex, lymph nodes, and midbrain; JADE3 in epididymis, placenta, and testis). In addition, the JADE family is conserved across species and has a target identity matching the mouse orthologue to the human sequences of $92.45 \%, 91.44 \%$, and $81.92 \%$, for JADE1, JADE2, and JADE3, respectively (25). Single-cell transcriptomic data from mouse kidneys revealed that all Jade proteins are expressed in the kidney with a predominance of Jade1 over Jade2 and Jade3 and with co-expression of all three proteins in cells of the collecting duct (Supplemental Fig. S1D) (26). Therefore, we decided to target Jade expression in this specific cell type.

Loss-of-function mutations in one Jade gene reveals independent expression of the remaining family members.

To analyze and compare transcriptional and translational consequences of Jade protein deficiency, we used the established mIMCD3 (murine inner medulla collecting duct 3 ) cell line and targeted individual Jade genes with CRISPR/Cas9. We designed two individual non-overlapping sgRNAs (single-guide RNAs) for each of the Jade genes to account for potential off-target effects. The first sgRNA was targeted to the region encoding the first PHD domain, the second sgRNA to the downstream exon containing the second PHD domain's start (Supplemental Fig. S2A). Mutant monoclonal cell lines were screened and validated by PCR and Sanger sequencing; the resulting mutant Jade protein structures are depicted in Supplemental Fig. S2B. When performing qPCR analyses for the different Jade genes, we observed a significant reduction of mRNA expression of the targeted Jade gene in the respective mutant cell line (Fig. 2A-C). Targeted proteomic PRM assays confirmed the absence of individual Jade protein 
expression in the specific Jade-deficient cell lines (Fig. 2D-F). Remarkably, we do not observe any compensatory upregulation of the remaining Jade family members on transcript and protein level.

\section{Proteomic analyses of Jade deficient cell lines reveal an increased abundance of almost all} proteasomal components.

We next performed unbiased proteomic analysis from all six Jade-deficient cell lines and the wildtype control. For each Jade-deficient cell line the principal component analysis showed the clustering of the replicates and the separation of WT versus the pair of mutant cell lines by the first principal component (Fig. 3A-C). When plotting the Student's T-test difference of KO1 versus KO2 for each of the Jade family members we found that they correlate and that expression of most deregulated proteins is shifted in the same direction within both mutant cell lines for each Jade protein (Supplemental Fig. S3A-C). Taking the sum of the significantly regulated proteins for both mutant cells for each Jade protein, we then calculated the overlap of significantly regulated proteins between the members of the Jade protein family (Fig. 3D). This revealed 644 proteins with significantly altered expression in all Jade1/2/3-deficient cell lines, supporting the concept that Jade protein family members share biological functions. A Gene Ontology and KEGG pathway analysis of these 644 proteins revealed terms related to the $26 \mathrm{~S}$ proteasome, ribosomes, and protein translation to be enriched (Fig. 3E). Indeed, many proteins related to the proteasome were significantly upregulated in the cell lines, as shown in a representative volcano blot of the Jade1 $\mathrm{KO} 2$ cell line (Fig. 3F) and in a heat-map summarizing the results from all cell lines (Fig. 3G). We also observed an altered expression of ribosomal proteins, tRNA ligases, as well as translation initiation and elongation factors in a similar manner (Supplemental Fig. S3D-F), being suggestive of a high protein turnover.

\section{Elevated proteasomal activity in Jade deficiency.}

To investigate whether these changes in protein expression caused a higher proteasomal activity, we monitored the degradation of a fluorogenic substrate for the chymotrypsin-like activity of the proteasome. Indeed, all Jade-deficient cell lines showed a significant increase in proteasomal activity as compared to the wildtype cells (Fig. 4 A). Consistently, using exogenously expressed Jade proteins, we could show that the proteasome activity is reduced compared to an overexpressed control protein (Fig. 4B).

Increased proteasome abundance is not caused by transcriptional regulation.

Based on the role of Jade1/2/3 as transcriptional and putative epigenetic regulators, we aimed to investigate whether the increased proteasome activity was caused by transcriptional upregulation of its components. To answer this question, we performed unbiased transcriptomic analyses with mRNA- 
Seq from all six Jade-deficient cell lines and the control cell line. For Jade1 deficient cell lines, principal component analysis showed a narrow clustering of the replicates and a clear separation of WT versus mutant cell lines accounting for $75 \%$ of the variance (Supplemental Fig. S4A). For Jade2 and Jade3 the replicates clustered clearly together, the separation from the WT cell lines was based primarily on the second principal component (Supplemental Fig. S4B,C). When plotting the fold change for KO1 versus KO2 for Jade1 we saw a clear correlation (Supplemental Fig. S4D). The same visualization for Jade2 and Jade3 revealed that the individual mutant cell lines for each of them are less correlated than seen for Jade1 (Supplemental Fig. S4E,F). Taking the sum of the significantly regulated genes for each Jade family member, we found that 567 genes were commonly regulated within the family (Supplemental Fig. S5A). However, when we individually checked the expression of the proteasomal components across all cell lines we did not observe any expression changes (Supplemental Fig. S5B,C). Additionally, we did not observe an upregulation in ribosomal genes and genes encoding for tRNA ligases or transcription initiation and elongation factors (Supplemental Fig. S5D-F). Taken together, the increased abundance and activity of the proteasome was not based on increased transcription in the Jade deficient cells.

\section{Proteasomal core components co-precipitate with Jade1L and Jade1S.}

Searching for a direct connection between the $26 \mathrm{~S}$ proteasome and Jade proteins, we re-expressed either Flag-tagged Jade1S or Jade1L or a control protein in a Jade1-deficient cell line and performed an anti-Flag coimmunoprecipitation experiment. Pulled-down protein complexes were analyzed by nLCMS/MS. The heatmap of iBAQs of the proteins that are exclusively found in the Jade1L/S samples shows Jade1 as most abundant among these proteins (Fig. 5A). Plotting the Student's T-test Difference between Jade1L and the control versus the -log Student's T-test $p$-value, we observed not only several known Jade1 interactors (green) but also several components of the proteasome (magenta) significantly enriched in the Jade1L sample (Fig. 5B). Moreover, we could confirm the same for Jade1S. Here, specifically Psma1 was among the most enriched proteins (Fig $5 \mathrm{C}$ ).

\section{Discussion}

JADE1 and in parts the other members of the JADE protein family have been previously described as components of several protein complexes and their potential cell biological functions have been mainly deduced by JADE interacting or associated proteins. Based on this, JADE proteins were found to be part of the HBO1/HAT complex (14), to regulate pVHL, beta-catenin and by that canonical WNT signaling on multiple levels $(7,9)(11)$, or to be involved in cell cycle regulation through a Plk1/CK1 kinase module $(27,28)$. In addition, altered expression of JADE proteins has been identified in several tumor entities, in line with their protein-protein interactions with several tumor suppressors, including 
pVHL and Ing4 $(6,24)$. Up to now, research on Jade proteins was primarily focused on the short isoform of JADE1 (JADE1S) (19) and not a single study has compared all three individual JADE proteins in the very same cellular context. In this study, we present for the first time an unbiased comparison of the proteomic and transcriptomic changes in Jade1, Jade2, and Jade3 deficient cell lines. Multiple previous studies suggested a specific role of JADE1 in renal ciliopathies (VHL, NPH $(6,7,11)$ ), ADPKD (12), renal cell carcinoma $(6,29,30)$ and kidney regeneration after injury (31). Strikingly, we could confirm some of the key protein-protein interactions (pVHL, NPHP1 and NPHP4) related to renal ciliopathies for all three Jade proteins (Fig. 1). Therefore, we continued our work with the well-characterized renal tubular epithelial cell line (mIMCD3; murine inner medullary collecting duct 3 ) to generate the Jade deficient cell lines for the at hand comparative study. Importantly, previously published datasets confirmed that renal epithelial cells of the collecting duct express all Jade proteins in vivo (Supplemental Fig. S1D).

With CRISPR/Cas9 mediated genome editing, we generated two independent Jade deficient cell lines for each Jade family member. These cell lines were validated by Sanger sequencing, qPCR analyses of mRNA expression, and a targeted proteomics approach (Fig. 2). Proteomic expression profiling revealed upregulated expression of more than $80 \%$ of the individual subunits of the $26 \mathrm{~S}$ proteasome (Fig. 3G) and we could demonstrate increased proteasome activity accordingly, underlining the functional relevance of the altered proteasome abundance. This suggests that JADE proteins have a common function in balancing or tuning proteasome abundance. Since this is not caused by transcriptional regulation of proteasome expression as reflected by our RNASeq analyses (Supplemental Fig. S4), we conclude, that Jade proteins might either modulate posttranslational modifications of individual proteasome components or, unspecifically, induce the process of proteophagy. At this point, the finding of proteasomal subunits co-precipitating with Jade1S and Jade1L strongly suggests a direct involvement of Jade proteins in the targeting of the proteasome for degradation. While the detailed underlying mechanism of this regulation still needs to be discovered, the observed high proteasomal activity in Jade deficient cells has several implications in the context of renal diseases. Enhanced proteasomal activity might increase the sensitivity of cells towards therapeutic inhibition of the proteasome. In Jade deficient cells, proteasome inhibition might more efficiently increase the unfolded protein burden and proteotoxic stress to toxic levels ultimately leading to apoptotic cell death. This could be relevant both for cancer cells with low or no Jade expression as well as in renal ciliopathies. Here, the disease genes pVHL, NPHP's and Polycystin-1 have been demonstrated to stabilize JADE1 expression $(1,12)$ with mutant proteins lacking this specific effect $(7,12)$. In consequence, the low levels of JADE in mutant cells could cause increased proteasomal activity. Remarkably, the use of the proteasome inhibitor Carfilzomib has been shown to ameliorate the cystic kidney phenotype in a mouse model of ADPLD (32) by increasing cell death and decreasing 
proliferation of renal epithelial cells. In this model, Jade deregulation and the general high proteasomal activity might be one of the mechanisms targeted by Carfilzomib. Proteasome inhibitors have also been very recently identified among other drugs in a study screening about 8000 compounds for their effect on viability of PKD deficient cells (33). Further preclinical studies in mouse models are required to investigate under which exact conditions targeting the proteasome in cystic kidney could be a useful option.

Jade1 is a protein localized at the ciliary transition zone and interacting with ciliopathy proteins, suggesting that the high abundance and activity of the proteasome might affect primary cilia. In all Jade deficient cell lines, we do not observe any significant change in the number of ciliated cells (data not shown). The UPS however, has been shown to be essential for the regulation of primary cilia. Inhibition of the proteasome with MG132 or depletion of proteasomal subunits with RNAi affects both ciliogenesis and ciliary disassembly (34). Interestingly, many proteasomal subunits have been identified as part of a protein complexes pulled-down with transitions zone and basal body proteins NPHP2, NPHP5 and NPHP8 (35) and with BBS proteins (36). Increased global proteasomal activity has not been directly linked to any ciliary function. One could speculate, that increased proteasomal activity as a result of reduced JADE expression might positively affect primary cilia in renal ciliopathies. Interestingly, loss of cilia has been found to suppress cyst growth in ADPKD (37). As such, loss of cilia may indeed be positive in some renal ciliopathies and might be counteracted under certain conditions through depletion of Jade and subsequent upregulation of proteasomal activity.

\section{Acknowledgements}

We thank Stefanie Keller and Martyna Brütting for excellent technical assistance and members of the laboratories for helpful discussions. In particular, we want to thank David Vilchez for his critical advice. We acknowledge the help of the CECAD proteomics core facility and the Cologne Center for Genomics, Cologne, Germany. We thank the FACS \& IMAGING Core Facility at Max Planck Institute for Biology of Ageing, Cologne, for assisting with sorting cells for generation of Jade KO cell lines. This study was supported by the German Research Foundation (SCHE1562/8-1 to BS). Roman-Ulrich Müller received support from the Ministry of Science North Rhine-Westphalia (Nachwuchsgruppen.NRW 2015-2021).

\section{Materials and Methods}

Plasmids and antibodies

270 Plasmids containing JADE1S, pVHL, and EPS ${ }^{1-225}$ CDNA were previously described (11, 38). JADE1L, 271 JADE2 and JADE3 CDNA were obtained by PCR from HEK293T cDNA. Jade1S, Jade1L, Jade2, and Jade3 272 cDNA were obtained by PCR from mIMCD3 cDNA. cDNA fragments were inserted into a modified 273 pcDNA6 vector (Invitrogen) containing an N-terminal FLAG-tag using standard cloning techniques. For 
the generation of the CRISPR/Cas9-mediated mutant cell lines the selected single guide (sg)RNAs were cloned into pSpCas9(BB)-2A-GFP (PX458), gifted from Feng Zhang (Addgene plasmid \#48138)(39). All plasmids were verified by Sanger sequencing. Primary antibodies were obtained from Sigma-Aldrich (monoclonal mouse anti-FLAG (M2), F3165, 1:10000; polyclonal rabbit anti-FLAG, F7425, 1:2000) and Serotec (monoclonal mouse anti-V5, MCA1360, 1:5000). Secondary antibodies were obtained from Jackson ImmunoResearch (goat antirabbit HRP-coupled, 111-035-003; goat anti-mouse HRP-coupled, 115-035-003; both 1:30000).

\section{Cell culture and transfections}

Murine inner-medullary collecting duct (mIMCD3, ATCC CRL-2123) cells were cultured in DMEM-F12 (Sigma-Aldrich) supplemented with $10 \%$ fetal bovine serum (FBS) and $2 \mathrm{mM}$ GlutaMAX (Gibco). For FACS sorted cell lines the medium was additionally supplemented with $100 \mathrm{U} / \mathrm{ml}$ PenicillinStreptomycin (Gibco). mIMCD3 subclone 8 (sc.\#8) was selected after FACS sorting the parental line into single cells for a higher genetic homogeneity. For the generation of the CRISPR/Cas9-mediated mIMCD3 sc.\#8 mutant cell lines, the cells were seeded in 6-well plates, grown to $50 \%$ confluency and transfected with Lipofectamine 2000 (Invitrogen) according to the manufacturer's instructions. HEK293T cells were cultured in DMEM (Gibco) supplemented with 10\% FBS. For transfection experiments in HEK293T cells, the cells were seeded in $10 \mathrm{~cm}$ dishes and grown to $50-60 \%$ confluency. Plasmid DNA was transiently transfected using the calcium phosphate method. $24 \mathrm{~h}$ after transfection cells were harvested in $8 \mathrm{ml}$ of cold PBS, centrifuged and the cell pellet was used for lysis and subsequent coimmunoprecipitation. All cells were cultured at $37^{\circ} \mathrm{C}$ in the presence of $5 \% \mathrm{CO}_{2}$ and tested negative for mycoplasmas.

\section{Coimmunoprecipitation}

HEK293T cell pellets were resuspended in $1 \mathrm{ml}$ of lysis buffer (20 mM Tris (pH 7.5), 1\% (v/v) TritonX$100,50 \mathrm{mM} \mathrm{NaCl}, 15 \mathrm{mM} \mathrm{Na}_{4} \mathrm{P}_{2} \mathrm{O}_{7}, 50 \mathrm{mM} \mathrm{NaF}$ ) and incubated for $15 \mathrm{~min}$ at $4{ }^{\circ} \mathrm{C}$. After homogenization the cell suspension was centrifuged $\left(20,000 \times \mathrm{g}, 15 \mathrm{~min}, 4{ }^{\circ} \mathrm{C}\right)$ and then the supernatant was ultracentrifuged $\left(100,000 \times \mathrm{g}, 30 \mathrm{~min}, 4^{\circ} \mathrm{C}\right) .50 \mu \mathrm{l}$ of the supernatant after ultracentrifugation was used as lysate (input) and boiled with 2x Laemmli buffer. Anti-FLAG agarose beads (M2, Sigma) were added to the remaining supernatant. After incubation for $1 \mathrm{~h}$ at $4{ }^{\circ} \mathrm{C}$ the beads were extensively washed with lysis buffer and boiled in Laemmli buffer. Proteins were resolved by $10 \%$ SDS-Page (NPHP1 and NPHP4 coimmunoprecipitations) and $12 \%$ SDS-Page (pVHL coimmunoprecipitations). 
Generation of mutant cell lines

For each member of the Jade family two sgRNAs were designed using the CRISPR finder tool (http://www.sanger.ac.uk/htgt/wge/)(40) and benchling (http://benchling.com). The sgRNA sequences are shown in supplemental table S1. Cloning of the sgRNA containing plasmids and transfection are described above. $48 \mathrm{~h}$ after transfection GFP-positive cells were sorted in 96-well plates on a BD FACSAriatnm III. After expanding the colonies arising from single cells, a first screening for mutants was performed using PCR, followed by Sanger sequencing. Primer sequences can be obtained from supplemental table S1. Validation was done by qPCR and a PRM assay.

\section{$q P C R$}

mIMCD3 wild type and Jade mutant cells were washed with PBS and RNA extraction was performed with the Direct-zol RNA Miniprep kit (Zymo Research) following the manufacturer's instructions including a DNase1 treatment step. This was followed by reverse transcription using the High-Capacity cDNA Reverse Transcription kit (Applied Biosystems). Jade1/2/3 mRNA was assessed by SYBR Green (Thermo Fisher Scientific) qPCR using Hprt1 as endogenous control. Primers are listed in supplemental table S1. The qPCR experiments were performed on a QuantStudio 12K Flex Real_time PCR System (Thermo Fisher Scientific). Data were analyzed using a student's t-test and are presented as mean +/standard error of the mean (SEM). Significance levels are indicated in the figure legends.

\section{PRM assays}

Jade protein expression has been difficult to detect by shotgun proteomics experiments. Therefore, we decided to use a parallel reaction monitoring (PRM) assay (41). Preparation of the samples for targeted mass spectrometry was performed as previously described (42). All samples were measured by the proteomics facility at CECAD. All samples were analyzed on an Orbitrap Exploris 480 mass spectrometer coupled to an EASY nLC 1200 UPLC (both Thermo Scientific). Peptides were loaded with solvent $A(0.1 \%$ formic acid in water) onto an in-house packed analytical column ( $30 \mathrm{~cm} \times 75 \mu \mathrm{m}$ I.D., filled with $2.7 \mu \mathrm{m}$ Poroshell EC120 C18 (Agilent)). Peptides were chromatographically separated at a constant flow rate of $300 \mathrm{nl} \mathrm{min}{ }^{-1}$ using with the following gradient: initial $4 \%$ solvent $B(0.1 \%$ formic acid in $80 \%$ acetonitrile), ramp to $25 \%$ B within $72 \mathrm{~min}$, to $55 \%$ B within 8 min and to $95 \%$ B within 2 min, followed by washing and column equilibration. The Exploris was equipped with a FAIMS Pro Interface (Thermo) set to a CV of -47 and operated in scheduled PRM mode. The MS1 survey scan was acquired from 400 to $1000 \mathrm{~m} / \mathrm{z}$ at a resolution of 120,000 . Target masses were isolated in a $2 \mathrm{~h}$ window and fragmented by HCD with collision energy of $30 \%$. The AGC target was set to $100 \%$ and resulting spectra recorded with a resolution of 120,000 . Product ions were detected in the Orbitrap at a resolution of 17,500 . 
Target list generation and follow-up analysis were performed in Skyline 20.2. Unique target peptides and their retention times were chosen based on their detectability in data-independent trials using the identical setup and gradient. Results were compared against their theoretical spectra simulated by the Prosit algorithm (43) plugin in Skyline $(44,45)$. Only areas with a dotp value above 0.7 were included in the analysis to ensure the confidence of the result. The targets are listed in supplemental table S2.

\section{Proteome analyses}

For each replicate, a $10 \mathrm{~cm}$ dish of mIMCD3 cells of the indicated genotype was harvested and snapfrozen. Pellets were resuspended in Urea buffer $(8 \mathrm{~m}$ Urea, $50 \mathrm{~mm}$ ammoniumbicarbonate, Halts Protease-phosphatase-inhibitor (Thermo Scientific)). After clearing of the sample (16000g, $30 \mathrm{~min}$ at $4^{\circ} \mathrm{C}$ ) the lysates were reduced ( $10 \mathrm{mM}$ Dithiothreitol, $1 \mathrm{~h}, \mathrm{RT}$ ) and alkylated (50 mM Chloroacetamide, $30 \mathrm{~min}, \mathrm{RT}$, in the dark). An equal amount of protein was diluted to reach a concentration of $2 \mathrm{M}$ urea and subjected to tryptic digestion (enzyme:substrate ratio of 1:50). On the next day, double-layered stage-tip clean up (C18) was performed.

All samples were analyzed on a Q Exactive Plus Orbitrap (Thermo Scientific) mass spectrometer that was coupled to an EASY nLC (Thermo Scientific). Peptides were loaded with solvent A ( $0.1 \%$ formic acid in water) onto an in-house packed analytical column (50 cm - $75 \mu \mathrm{m}$ I.D., filled with $2.7 \mu \mathrm{m}$ Poroshell EC120 C18, Agilent). Peptides were chromatographically separated at a constant flow rate of 250 $\mathrm{nL} / \mathrm{min}$ using the following gradient: $3-4 \%$ solvent $\mathrm{B}$ (0.1\% formic acid in $80 \%$ acetonitrile) within 1.0 min, 4-27\% solvent B within 119.0 min, 27-50\% solvent B within 19.0 min, 50-95\% solvent B within 1.0 min, followed by washing and column equilibration. The mass spectrometer was operated in datadependent acquisition mode. The MS1 survey scan was acquired from $300-1750 \mathrm{~m} / \mathrm{z}$ at a resolution of 70,000. The top 10 most abundant peptides were isolated within a 1.8 Th window and subjected to HCD fragmentation at a normalized collision energy of $27 \%$. The AGC target was set to $5 \mathrm{e} 5$ charges, allowing a maximum injection time of $55 \mathrm{~ms}$. Product ions were detected in the Orbitrap at a resolution of 17,500 . Precursors were dynamically excluded for $30.0 \mathrm{~s}$.

All mass spectrometric raw data were processed with Maxquant (version 1.5.3.8) using default parameters. Briefly, MS2 spectra were searched against the canonical Uniprot mouse fasta database (UP000000589; May 4, 2020) and the MaxQuant default list of common contaminants. False discovery rates on protein and PSM level were estimated by the target-decoy approach to 1\% (Protein FDR) and 1\% (PSM FDR) respectively. The minimal peptide length was set to 7 amino acids and carbamidomethylation at cysteine residues was considered as a fixed modification. Oxidation (M) and Acetyl (Protein $\mathrm{N}$-term) were included as variable modifications. The match-between runs option was enabled. Student's T-tests were calculated in Perseus (version 1.6.1.1) after removal of decoys and potential contaminants. Data were filtered for at least 3 out of 3 values in at least one condition. 
Remaining missing values were imputed with random values from the left end of the intensity distribution using Perseus defaults.

Interactome analysis

For each replicate, a $10 \mathrm{~cm}$ dish of Jade1 KO1 mIMCD3 cells was transfected with $10 \mu \mathrm{g}$ of either Flagtagged Jade1S, Jade1L or an empty vector control using Lipofectamine (Invitrogen) 2000 following the manufacture's instructions. $48 \mathrm{~h}$ after transfection cells were harvested and lyzed (50 mM Tris (pH 7.5), $150 \mathrm{mM} \mathrm{NaCl}, 0.5 \%(\mathrm{w} / \mathrm{v})$ Sodium deoxycholate, 1\% (v/v) Triton X-100) for 15 min @ $4^{\circ} \mathrm{C}$. After sonication (Bioruptor, $10 \mathrm{~min}$, cycle $30 / 30 \mathrm{sec}$ ) and centrifugation $\left(4^{\circ} \mathrm{C}, 15 \mathrm{~min}\right.$ ) anti-FLAG agarose beads (M2, Sigma) were added to the supernatant and incubated at $4^{\circ} \mathrm{C}$ overnight. After extensive washing of the beads, $5 \%$ SDS in 1xPBS was added and proteins were released from the beads by incubation at $95^{\circ} \mathrm{C}$ for $3 \mathrm{~min}$. Subsequently, the supernatant was reduced with DTT and alkylated with CAA followed by two single-pot solid-phase-enhanced sample preparations (SP3).

All samples were analyzed on a Q Exactive Plus Orbitrap (Thermo Scientific) mass spectrometer that was coupled to an EASY nLC (Thermo Scientific). Peptides were loaded with solvent A ( $0.1 \%$ formic acid in water) onto an in-house packed analytical column (50 cm - $75 \mu \mathrm{m}$ I.D., filled with $2.7 \mu \mathrm{m}$ Poroshell EC120 C18, Agilent). Peptides were chromatographically separated at a constant flow rate of 250 $\mathrm{nL} / \mathrm{min}$ using the following gradient: $3-5 \%$ solvent $\mathrm{B}$ (0.1\% formic acid in $80 \%$ acetonitrile) within 1.0 min, $5-30 \%$ solvent B within 65.0 min, $30-50 \%$ solvent B within 13.0 min, 50-95\% solvent B within 1.0 min, followed by washing and column equilibration. The mass spectrometer was operated in datadependent acquisition mode. The MS1 survey scan was acquired from 300-1750 m/z at a resolution of 70,000. The top 10 most abundant peptides were isolated within a 1.8 Th window and subjected to HCD fragmentation at a normalized collision energy of $27 \%$. The AGC target was set to 5 e5 charges, allowing a maximum injection time of $110 \mathrm{~ms}$. Product ions were detected in the Orbitrap at a resolution of 35,000 . Precursors were dynamically excluded for $15.0 \mathrm{~s}$.

All mass spectrometric raw data were processed with Maxquant (version 1.5.3.8) using default parameters. Briefly, MS2 spectra were searched against the canonical Uniprot MOUSE_UP000000589.fasta (downloaded at: 26.08.2020) database, including a list of common contaminants. False discovery rates on protein and PSM level were estimated by the target-decoy approach to 1\% (Protein FDR) and 1\% (PSM FDR) respectively. The minimal peptide length was set to 7 amino acids and carbamidomethylation at cysteine residues was considered as a fixed modification. Oxidation (M) and Acetyl (Protein N-term) were included as variable modifications. The matchbetween runs option was enabled for replicates from the same group. LFQ quantification was enabled using default settings. 
In vitro assay of 265 proteasome activity

The proteasomal activity assay was performed as previously described (46). For each replicate with the mIMCD3 cells, the cells were grown into 6-well plates and grown until 70\% confluency. HEK29T cells were grown into 6-well plates, transfected at 50\% confluency with $2 \mu \mathrm{g}$ of DNA using the calcium phosphate method, and harvested $24 \mathrm{~h}$ after transfection. Cells were collected on proteasomal activity assay buffer (50 mM Tris-HCL, pH 7.5, 10\% glycerol, 5 mM MgCl $2,0.5$ mM EDTA, 2 mM ATP, 1 mM DTT) and immediately frozen in liquid nitrogen. Before performing the assay, samples were lyzed and centrifuged at $10000 \mathrm{~g}$ for $10 \mathrm{~min}$ at $4^{\circ} \mathrm{C} .25 \mu \mathrm{g}$ of protein was loaded on a flat-bottom, black fluorescence 96-well microplate and incubated with the fluorogenic substrate (Z-Gly-Gly-Leu-AMC, BML-ZW8505-0005, ENZO Life Science). Fluorescence (360 nm excitation, $430 \mathrm{~nm}$ emission) was monitored every $5 \mathrm{~min}$ for $2 \mathrm{~h}$ at $37^{\circ} \mathrm{C}$ using a fluorescent plate reader (EnSpire Multimode Plate Reader, Perkin Elmer). Samples were assayed in technical triplicates and in biological replicates as indicated in the figure legend.

\section{mRNA sequencing}

Total RNA extraction was performed with the direct-zol RNA Miniprep kit (Zymo Research) following the manufacture's instructions. Library preparation and sequencing was performed by the Cologne Center for Genomics. Libraries were prepared using the Illumina ${ }^{\circledR}$ TruSeq $^{\circledR}$ mRNA stranded sample preparation Kit. Library preparation started with $300 \mathrm{ng}$ total RNA. After poly-A selection, mRNA was purified and fragmented using divalent cations under elevated temperature. The RNA fragments underwent reverse transcription using random primers, followed by second strand CDNA synthesis with DNA Polymerase I and RNase H. After end repair and A-tailing, indexing adapters were ligated. The products were then purified and amplified to create the final cDNA libraries. After library validation and quantification (TapStation 4200, Agilent), equimolar amounts of library were pooled and quantified by using the Peqlab KAPA Library Quantification Kit and the Applied Biosystems 7900HT Sequence Detection System. The pool was sequenced on an Illumina NovaSeq6000 with PE100 read length and a minimum of 35 million reads per sample.

The reads were trimmed with Trimmomatic version 0.36 (47) using default parameters. The trimmed reads were mapped to the $\mathrm{GRCm} 39$ mouse reference genome with STAR version 2.6 (48) using default parameters. mRNA expression was analyzed using DESeq2 (version 1.28.1)(49) package of $R$ (version 4.0.0) software (https://www.R-project.org/). A p-value cut-off of $<0.05$ was applied to the list of differentially regulated mRNAs after pairwise comparison of the Jade deficient cell lines with wild type. P-values were adjusted for multiple testing. 
Raw data deposition

The proteome and interactome mass spectrometry proteomics data have been deposited to the ProteomeXchange Consortium (http://proteomecentral.proteomexchange.org) via the PRIDE partner repository (50) with the dataset identifier PXD028800. RNA sequencing data have been deposited in the ArrayExpress database at EMBL-EBI (www.ebi.ac.uk/arrayexpress) under accession number EMTAB-11021 (51). Login information are available upon request.

\section{References}

1. Zhou, M. I., Wang, H., Ross, J. J., Kuzmin, I., Xu, C., and Cohen, H. T. (2002) The von HippelLindau tumor suppressor stabilizes novel plant homeodomain protein Jade-1. J. Biol. Chem. 277, 39887-39898

2. Foster, K., Prowse, A., van den Berg, A., Fleming, S., Hulsbeek, M. M., Crossey, P. A., Richards, F. M., Cairns, P., Affara, N. A., and Ferguson-Smith, M. A. (1994) Somatic mutations of the von Hippel-Lindau disease tumour suppressor gene in non-familial clear cell renal carcinoma. Hum Mol Genet. 3, 2169-2173

3. Gnarra, J. R., Tory, K., Weng, Y., Schmidt, L., Wei, M. H., Li, H., Latif, F., Liu, S., Chen, F., and Duh, F. M. (1994) Mutations of the VHL tumour suppressor gene in renal carcinoma. Nat Genet. 7, 85-90

4. Shuin, T., Kondo, K., Torigoe, S., Kishida, T., Kubota, Y., Hosaka, M., Nagashima, Y., Kitamura, H., Latif, F., and Zbar, B. (1994) Frequent somatic mutations and loss of heterozygosity of the von Hippel-Lindau tumor suppressor gene in primary human renal cell carcinomas. Cancer Res. 54, 2852-2855

5. Whaley, J. M., Naglich, J., Gelbert, L., Hsia, Y. E., Lamiell, J. M., Green, J. S., Collins, D., Neumann, H. P. H., Laidlaw, J., Li, F. P., Klein-Szanto, A. J. P., Seizinger, B. R., and Kley, N. (1994) Germ-Line Mutations in the von Hippel-Lindau Tumor-Suppressor Gene Are Similar to Somatic von Hippel-Lindau Aberrations in Sporadic Renal Cell Carcinoma. Am J Hum Genet. 55, 10921102

6. Zeng, L., Bai, M., Mittal, A. K., El-Jouni, W., Zhou, J., Cohen, D. M., Zhou, M. I., and Cohen, H. T. (2013) Candidate tumor suppressor and pVHL partner Jade-1 binds and inhibits AKT in renal cell carcinoma. Cancer Res. 73, 5371-5380

7. Zhou, M. I., Wang, H., Foy, R. L., Ross, J. J., and Cohen, H. T. (2004) Tumor suppressor von Hippel-Lindau (VHL) stabilization of Jade-1 protein occurs through plant homeodomains and is VHL mutation dependent. Cancer Res. 64, 1278-1286

8. Zhou, M. I., Foy, R. L., Chitalia, V. C., Zhao, J., Panchenko, M. V., Wang, H., and Cohen, H. T. (2005) Jade-1, a candidate renal tumor suppressor that promotes apoptosis. Proc. Natl. Acad. Sci. U.S.A. 102, 11035-11040

9. Chitalia, V. C., Foy, R. L., Bachschmid, M. M., Zeng, L., Panchenko, M. V., Zhou, M. I., Bharti, A., Seldin, D. C., Lecker, S. H., Dominguez, I., and Cohen, H. T. (2008) Jade-1 inhibits Wnt signalling by ubiquitylating beta-catenin and mediates Wnt pathway inhibition by pVHL. Nat. Cell Biol. 10, 1208-1216

10. Benzing, T., and Schermer, B. (2012) Clinical spectrum and pathogenesis of nephronophthisis. Current Opinion in Nephrology and Hypertension. 21, 272-278

11. Borgal, L., Habbig, S., Hatzold, J., Liebau, M. C., Dafinger, C., Sacarea, I., Hammerschmidt, M., Benzing, T., and Schermer, B. (2012) The ciliary protein nephrocystin-4 translocates the canonical Wnt regulator Jade- 1 to the nucleus to negatively regulate $\beta$-catenin signaling. J. Biol. Chem. 287, 25370-25380

12. Foy, R. L., Chitalia, V. C., Panchenko, M. V., Zeng, L., Lopez, D., Lee, J. W., Rana, S. V., Boletta, A., Qian, F., Tsiokas, L., Piontek, K. B., Germino, G. G., Zhou, M. I., and Cohen, H. T. (2012) 
Polycystin-1 regulates the stability and ubiquitination of transcription factor Jade-1. Hum. Mol. Genet. 21, 5456-5471

13. Hildebrandt, F., Benzing, T., and Katsanis, N. (2011) Ciliopathies. N Engl J Med. 364, 1533-1543

14. Foy, R. L., Song, I. Y., Chitalia, V. C., Cohen, H. T., Saksouk, N., Cayrou, C., Vaziri, C., Côté, J., and Panchenko, M. V. (2008) Role of Jade-1 in the histone acetyltransferase (HAT) HBO1 complex. J. Biol. Chem. 283, 28817-28826

15. Panchenko, M. V., Zhou, M. I., and Cohen, H. T. (2004) von Hippel-Lindau partner Jade-1 is a transcriptional co-activator associated with histone acetyltransferase activity. J. Biol. Chem. 279, 56032-56041

16. Johmura, Y., Osada, S., Nishizuka, M., and Imagawa, M. (2008) FAD24 acts in concert with histone acetyltransferase $\mathrm{HBO} 1$ to promote adipogenesis by controlling DNA replication. $J$ Biol Chem. 283, 2265-2274

17. Quintela, M., Sieglaff, D. H., Gazze, A. S., Zhang, A., Gonzalez, D., Francis, L., Webb, P., and Conlan, R. S. (2019) HBO1 directs histone H4 specific acetylation, potentiating mechanotransduction pathways and membrane elasticity in ovarian cancer cells. Nanomedicine. 17, 254-265

18. Tzouanacou, E., Tweedie, S., and Wilson, V. (2003) Identification of Jade1, a gene encoding a PHD zinc finger protein, in a gene trap mutagenesis screen for genes involved in anteroposterior axis development. Mol Cell Biol. 23, 8553-8552

19. Panchenko, M. V. (2016) Structure, function and regulation of jade family PHD finger 1 (JADE1). Gene. 589, 1-11

20. Han, X., Gui, B., Xiong, C., Zhao, L., Liang, J., Sun, L., Yang, X., Yu, W., Si, W., Yan, R., Yi, X., Zhang, D., Li, W., Li, L., Yang, J., Wang, Y., Sun, Y. E., Zhang, D., Meng, A., and Shang, Y. (2014) Destabilizing LSD1 by Jade-2 promotes neurogenesis: an antibraking system in neural development. Mol. Cell. 55, 482-494

21. Wu, C.-Y., Persaud, S. D., and Wei, L.-N. (2016) Retinoic Acid Induces Ubiquitination-Resistant RIP140/LSD1 Complex to Fine-Tune Pax6 Gene in Neuronal Differentiation. Stem Cells. 34, 114123

22. Anan, K., Hino, S., Shimizu, N., Sakamoto, A., Nagaoka, K., Takase, R., Kohrogi, K., Araki, H., Hino, Y., Usuki, S., Oki, S., Tanaka, H., Nakamura, K., Endo, F., and Nakao, M. (2018) LSD1 mediates metabolic reprogramming by glucocorticoids during myogenic differentiation. Nucleic Acids Res. 46, 5441-5454

23. Jian, Y., Wang, M., Zhang, Y., Ou, R., Zhu, Z., Ou, Y., Chen, X., Liang, X., Ding, Y., Song, L., Xu, X., and Liao, W. (2018) Jade family PHD finger 3 (JADE3) increases cancer stem cell-like properties and tumorigenicity in colon cancer. Cancer Letters. 428, 1-11

24. Doyon, Y., Cayrou, C., Ullah, M., Landry, A.-J., Côté, V., Selleck, W., Lane, W. S., Tan, S., Yang, X.J., and Côté, J. (2006) ING tumor suppressor proteins are critical regulators of chromatin acetylation required for genome expression and perpetuation. Mol Cell. 21, 51-64

25. Yates, A. D., Achuthan, P., Akanni, W., Allen, J., Allen, J., Alvarez-Jarreta, J., Amode, M. R., Armean, I. M., Azov, A. G., Bennett, R., Bhai, J., Billis, K., Boddu, S., Marugán, J. C., Cummins, C., Davidson, C., Dodiya, K., Fatima, R., Gall, A., Giron, C. G., Gil, L., Grego, T., Haggerty, L., Haskell, E., Hourlier, T., Izuogu, O. G., Janacek, S. H., Juettemann, T., Kay, M., Lavidas, I., Le, T., Lemos, D., Martinez, J. G., Maurel, T., McDowall, M., McMahon, A., Mohanan, S., Moore, B., Nuhn, M., Oheh, D. N., Parker, A., Parton, A., Patricio, M., Sakthivel, M. P., Abdul Salam, A. I., Schmitt, B. M., Schuilenburg, H., Sheppard, D., Sycheva, M., Szuba, M., Taylor, K., Thormann, A., Threadgold, G., Vullo, A., Walts, B., Winterbottom, A., Zadissa, A., Chakiachvili, M., Flint, B., Frankish, A., Hunt, S. E., Ilsley, G., Kostadima, M., Langridge, N., Loveland, J. E., Martin, F. J., Morales, J., Mudge, J. M., Muffato, M., Perry, E., Ruffier, M., Trevanion, S. J., Cunningham, F., Howe, K. L., Zerbino, D. R., and Flicek, P. (2020) Ensembl 2020. Nucleic Acids Research. 48, D682-D688 
26. Park, J., Shrestha, R., Qiu, C., Kondo, A., Huang, S., Werth, M., Li, M., Barasch, J., and Suszták, K. (2018) Single-cell transcriptomics of the mouse kidney reveals potential cellular targets of kidney disease. Science. 360, 758-763

27. Borgal, L., Rinschen, M. M., Dafinger, C., Hoff, S., Reinert, M. J., Lamkemeyer, T., Lienkamp, S. S., Benzing, T., and Schermer, B. (2014) Casein kinase $1 \alpha$ phosphorylates the Wnt regulator Jade-1 and modulates its activity. J. Biol. Chem. 289, 26344-26356

28. Borgal, L., Rinschen, M. M., Dafinger, C., Liebrecht, V. I., Abken, H., Benzing, T., and Schermer, B. (2016) Jade-1S phosphorylation induced by CK1 $\alpha$ contributes to cell cycle progression. Cell Cycle. 15, 1034-1045

29. Lian, X., Duan, X., Wu, X., Li, C., Chen, S., Wang, S., Cai, Y., and Weng, Z. (2012) Expression and clinical significance of von Hippel-Lindau downstream genes: Jade- 1 and $\beta$-catenin related to renal cell carcinoma. Urology. 80, 485.e7-13

30. Xiao-Fen, W., Ting, C., Jie, L., Deng-Yang, M., Qing-Feng, Z., and Xin, L. (2016) Correlation analysis of VHL and Jade-1 gene expression in human renal cell carcinoma. Open Med (Wars). 11, 226-230

31. Havasi, A., Haegele, J. A., Gall, J. M., Blackmon, S., Ichimura, T., Bonegio, R. G., and Panchenko, M. V. (2013) Histone acetyl transferase (HAT) HBO1 and JADE1 in epithelial cell regeneration. Am. J. Pathol. 182, 152-162

32. Fedeles, S. V., Tian, X., Gallagher, A.-R., Mitobe, M., Nishio, S., Lee, S. H., Cai, Y., Geng, L., Crews, C. M., and Somlo, S. (2011) A genetic interaction network of five genes for human polycystic kidney and liver diseases defines polycystin- 1 as the central determinant of cyst formation. Nat Genet. 43, 639-647

33. Asawa, R. R., Danchik, C., Zahkarov, A., Chen, Y., Voss, T., Jadhav, A., Wallace, D. P., Trott, J. F., Weiss, R. H., Simeonov, A., and Martinez, N. J. (2020) A high-throughput screening platform for Polycystic Kidney Disease (PKD) drug repurposing utilizing murine and human ADPKD cells. Scientific reports. 10, 4203

34. Kim, J. H., Ki, S. M., Joung, J.-G., Scott, E., Heynen-Genel, S., Aza-Blanc, P., Kwon, C. H., Kim, J., Gleeson, J. G., and Lee, J. E. (2016) Genome-wide screen identifies novel machineries required for both ciliogenesis and cell cycle arrest upon serum starvation. Biochim Biophys Acta. 1863, 1307-1318

35. Sang, L., Miller, J. J., Corbit, K. C., Giles, R. H., Brauer, M. J., Otto, E. A., Baye, L. M., Wen, X., Scales, S. J., Kwong, M., Huntzicker, E. G., Sfakianos, M. K., Sandoval, W., Bazan, J. F., Kulkarni, P., Garcia-Gonzalo, F. R., Seol, A. D., O'Toole, J. F., Held, S., Reutter, H. M., Lane, W. S., Rafiq, M. A., Noor, A., Ansar, M., Devi, A. R. R., Sheffield, V. C., Slusarski, D. C., Vincent, J. B., Doherty, D. A., Hildebrandt, F., Reiter, J. F., and Jackson, P. K. (2011) Mapping the NephronophthisisJoubert-Meckel-Gruber Protein Network Reveals Ciliopathy Disease Genes and Pathways. Cell. 145, 513-528

36. Liu, Y. P., Tsai, I.-C., Morleo, M., Oh, E. C., Leitch, C. C., Massa, F., Lee, B.-H., Parker, D. S., Finley, D., Zaghloul, N. A., Franco, B., and Katsanis, N. (2014) Ciliopathy proteins regulate paracrine signaling by modulating proteasomal degradation of mediators. J Clin Invest. 124, 2059-2070

37. Ma, M., Tian, X., Igarashi, P., Pazour, G. J., and Somlo, S. (2013) Loss of cilia suppresses cyst growth in genetic models of autosomal dominant polycystic kidney disease. Nat Genet. 45, 1004-1012

38. Schermer, B., Ghenoiu, C., Bartram, M., Müller, R. U., Kotsis, F., Höhne, M., Kühn, W., Rapka, M., Nitschke, R., Zentgraf, H., Fliegauf, M., Omran, H., Walz, G., and Benzing, T. (2006) The von Hippel-Lindau tumor suppressor protein controls ciliogenesis by orienting microtubule growth. J Cell Biol. 175, 547-554

39. Ran, F. A., Hsu, P. D., Wright, J., Agarwala, V., Scott, D. A., and Zhang, F. (2013) Genome engineering using the CRISPR-Cas9 system. Nature Protocols. 8, 2281-2308

40. Hodgkins, A., Farne, A., Perera, S., Grego, T., Parry-Smith, D. J., Skarnes, W. C., and Iyer, V. (2015) WGE: a CRISPR database for genome engineering. Bioinformatics. 31, 3078-3080 
41. Rauniyar, N. (2015) Parallel Reaction Monitoring: A Targeted Experiment Performed Using High Resolution and High Mass Accuracy Mass Spectrometry. Int J Mol Sci. 16, 28566-28581

42. Bartram, M. P., Habbig, S., Pahmeyer, C., Höhne, M., Weber, L. T., Thiele, H., Altmüller, J., Kottoor, N., Wenzel, A., Krueger, M., Schermer, B., Benzing, T., Rinschen, M. M., and Beck, B. B. (2016) Three-layered proteomic characterization of a novel ACTN4 mutation unravels its pathogenic potential in FSGS. Hum Mol Genet. 25, 1152-1164

43. Gessulat, S., Schmidt, T., Zolg, D. P., Samaras, P., Schnatbaum, K., Zerweck, J., Knaute, T., Rechenberger, J., Delanghe, B., Huhmer, A., Reimer, U., Ehrlich, H.-C., Aiche, S., Kuster, B., and Wilhelm, M. (2019) Prosit: proteome-wide prediction of peptide tandem mass spectra by deep learning. Nat Methods. 16, 509-518

44. MacLean, B., Tomazela, D. M., Shulman, N., Chambers, M., Finney, G. L., Frewen, B., Kern, R., Tabb, D. L., Liebler, D. C., and MacCoss, M. J. (2010) Skyline: an open source document editor for creating and analyzing targeted proteomics experiments. Bioinformatics. 26, 966-968

45. Pino, L. K., Searle, B. C., Bollinger, J. G., Nunn, B., MacLean, B., and MacCoss, M. J. (2020) The Skyline ecosystem: Informatics for quantitative mass spectrometry proteomics. Mass Spectrom Rev. 39, 229-244

46. Kisselev, A. F., and Goldberg, A. L. (2005) Monitoring Activity and Inhibition of 26S Proteasomes with Fluorogenic Peptide Substrates. in Methods in Enzymology, pp. 364-378, Ubiquitin and Protein Degradation, Part A, Academic Press, 398, 364-378

47. Bolger, A. M., Lohse, M., and Usadel, B. (2014) Trimmomatic: a flexible trimmer for Illumina sequence data. Bioinformatics. 30, 2114-2120

48. Dobin, A., Davis, C. A., Schlesinger, F., Drenkow, J., Zaleski, C., Jha, S., Batut, P., Chaisson, M., and Gingeras, T. R. (2013) STAR: ultrafast universal RNA-seq aligner. Bioinformatics. 29, 15-21

49. Love, M. I., Huber, W., and Anders, S. (2014) Moderated estimation of fold change and dispersion for RNA-seq data with DESeq2. Genome Biology. 15, 550

50. Perez-Riverol, Y., Csordas, A., Bai, J., Bernal-Llinares, M., Hewapathirana, S., Kundu, D. J., Inuganti, A., Griss, J., Mayer, G., Eisenacher, M., Pérez, E., Uszkoreit, J., Pfeuffer, J., Sachsenberg, T., Yilmaz, S., Tiwary, S., Cox, J., Audain, E., Walzer, M., Jarnuczak, A. F., Ternent, T., Brazma, A., and Vizcaíno, J. A. (2019) The PRIDE database and related tools and resources in 2019: improving support for quantification data. Nucleic Acids Res. 47, D442-D450

51. Athar, A., Füllgrabe, A., George, N., Iqbal, H., Huerta, L., Ali, A., Snow, C., Fonseca, N. A., Petryszak, R., Papatheodorou, I., Sarkans, U., and Brazma, A. (2019) ArrayExpress update - from bulk to single-cell expression data. Nucleic Acids Research. 47, D711-D715

52. Rice, P., Longden, I., and Bleasby, A. (2000) EMBOSS: the European Molecular Biology Open Software Suite. Trends Genet. 16, 276-277

53. Letunic, I., and Bork, P. (2018) 20 years of the SMART protein domain annotation resource. Nucleic Acids Res. 46, D493-D496

54. Oughtred, R., Rust, J., Chang, C., Breitkreutz, B.-J., Stark, C., Willems, A., Boucher, L., Leung, G., Kolas, N., Zhang, F., Dolma, S., Coulombe-Huntington, J., Chatr-Aryamontri, A., Dolinski, K., and Tyers, M. (2021) The BioGRID database: A comprehensive biomedical resource of curated protein, genetic, and chemical interactions. Protein Sci. 30, 187-200

55. Consortium, T. U. (2019) UniProt: a worldwide hub of protein knowledge. Nucleic Acids Res. 47, D506-D515

56. Madeira F, Park Ym, Lee J, Buso N, Gur T, Madhusoodanan N, Basutkar P, Tivey Arn, Potter Sc, Finn Rd, and Lopez R (2019) The EMBL-EBI search and sequence analysis tools APIs in 2019. Nucleic Acids Res. 47, W636-W641

57. Uhlén, M., Fagerberg, L., Hallström, B. M., Lindskog, C., Oksvold, P., Mardinoglu, A., Sivertsson, Å., Kampf, C., Sjöstedt, E., Asplund, A., Olsson, I., Edlund, K., Lundberg, E., Navani, S., Szigyarto, C. A.-K., Odeberg, J., Djureinovic, D., Takanen, J. O., Hober, S., Alm, T., Edqvist, P.-H., Berling, H., Tegel, H., Mulder, J., Rockberg, J., Nilsson, P., Schwenk, J. M., Hamsten, M., Feilitzen, K. von, Forsberg, M., Persson, L., Johansson, F., Zwahlen, M., Heijne, G. von, Nielsen, J., and Pontén, F. (2015) Tissue-based map of the human proteome. Science. 10.1126/science.1260419 
650

651

652

653

654

655

656

657

658

659

660

661

662

663

664

665

666

667

668

669

670

671

672

673

674

675

676

677

678

679

680

681

682

683

684

Figure legends

Figure 1: All JADE protein family members co-precipitate with the ciliopathy proteins NPHP1, NPHP4, and pVHL. (A) Domain structure of the human JADE proteins. PHD - Cys4HisCys3 plant homeo domain; NLS - nuclear localization signal; PEST - protein degradation amino acid sequence. Domain information obtained from EMBOSS/epestfind (52), SMART(53), and NLS prediction (consensus sequence $K-(K / R)$ $-\mathrm{X}-(\mathrm{K} / \mathrm{R})$ ). (B-D) HEK293T cells were transiently transfected with (B) V5-tagged pVHL (V5.VHL), (C) V5-tagged Nphp4 (V5.NPHP4), and (D) V5-tagged Nphp1 (V5.NHPH1), and either FLAG-tagged JADE1S (F.JADE1S), Jade2 (F.JADE2), Jade3 (F.JADE3) or a control protein (F.EPS ${ }^{1-225}$ ). Immunoprecipitates with an anti-FLAG antibody were analyzed by immunoblotting with antibodies (anti-V5; anti-FLAG) as indicated. V5.VHL, V5.NPHP1, and V5.NPHP4 all coimmunoprecipitate with F.Jade1S/2/3 but not with F.EPS ${ }^{1-225}$. Blots are representative for at least three independent experiments. (E,F) HEK293T cells were transiently cotransfected with (E) V5.JADE1S and FLAG.JADE1S or (F) V5.JADE2 and FLAG.JADE2. FLAG.pVHL and FLAG.EPS ${ }^{1-225}$ served as positive and negative control, respectively. Immunoprecipitates with an anti-FLAG antibody were analyzed by immunoblotting with antibodies as indicated. V5.JADE1S and V5.JADE2 both coimmunoprecipitate with the respective FLAG-tagged JADE protein. (G) HEK293T cells were transiently transfected with V5.JADE2 and either FLAG-tagged JADE1S, JADE3, pVHL, or EPS ${ }^{1-225}$. Immunoprecipitates with an anti-FLAG antibody were analyzed by immunoblotting with antibodies (anti-V5; anti-FLAG) as indicated. V5.JADE2 coimmunoprecipitate with F.JADE1S, F.JADE3, and F.VHL but not with F.EPS ${ }^{1-225}$ (h.c.: heavy chain, l.c.: light chain).

Figure 2: Deletion of one specific Jade protein does affect neither transcription nor expression of the remaining family members. (A-C) qPCR data comparing the expression fold change of each targeted gene across the different cell lines. Only the expression of the targeted gene in the respective mutant cell lines is significantly reduced, while the other Jade mRNAs are not significantly altered. $(\mathrm{N}=3$, students t-test, $* p \leq 0.05, * * p \leq 0.01, * * * p \leq 0.001$ ) (D-F) Parallel reaction monitoring (PRM) assay for (D) Jade1, (E) Jade2, and (F) Jade3 comparing the peak areas for the selected peptides across mIMCD3 WT and Jade mutant cell lines for protein quantification. The localization of peptides selected for PRM assay is depicted above the bar charts. ( $N=3$ )

Figure 3: Proteomic analysis reveals upregulation of the proteasome in cell lines lacking either one of the Jade family members. (A-C) Principal component analysis (PCA) plot of the protein expression data of WT, Jade1 (A), Jade2 (B), and Jade3 (C) KO1 and KO2 cell lines. Depicted are the first two principal components (PC1 and PC2). The axes represent the percentages of variation explained by the principal components. The replicates of the different groups cluster together and the difference between mutant and wildtype groups explains the largest variance. (D) Venn diagram depicting the overlap between significantly regulated proteins across the Jade-deficient cell lines. The Jade1 section includes all proteins that are significantly regulated $(p$-value $<0.05)$ in either one or both of the Jade1- 
685

686

687

688

689

690

691

692

693

694

695

696

697

698

699

700

701

702

703

704

705

706

707

708

709

710

711

712

deficient cell lines. The Jade2 and Jade3 sections generated in the same way. (E) GO and KEGG pathway annotation based on a Fisher exact test of the 644 proteins found to be regulated in all Jade mutant cell lines. (F) Representative scatter plot with the t-test differences in protein expression of the Jade1 KO2 mutant cells vs the wildtype cells on the $\mathrm{x}$-axis and the statistical significance (- $\log _{10}$ Student's ttest $p$-value) on the $y$-axis. Proteins associated with the ubiquitin proteasome system are highlighted in magenta. (G) Heatmap of the proteins highlighted in (F) based on logarithmized LFQ values for all Jade-deficient cell lines and the control line visualizing the upregulation of proteasome-associated proteins.

Figure 4: Increased proteasome activity observed in Jade-deficient cell lines. (A) Measurement of the proteasome activity in wildtype and Jade-deficient mIMCD3 cell lines. The proteasome activity of Jade1, Jade2, or Jade3-deficient cell lines is increased compared to the wildtype control. Quantification of the slope as readout for proteasome activity ( $N=3$, one-way ANOVA with Dunnett's post-hoc test, $* * * * p \leq 0.0001$ ) (B) Measurement of the proteasome activity in HEK293T cell with either Jade1S, Jade1L, Jade2, Jade3, or Podocin (control) overexpressed. All cell lines with overexpressed Jade proteins show a decreased activity compared to the control with only Jade1S/L reaching statistical significance. Quantification of the slope as readout for proteasome activity ( $N=3$, one-way ANOVA Dunnett's post-hoc test, * $p \leq 0.05$ )

Figure 5: Interactome analysis reveals a link between Jade1L/S proteins and proteasomal components. (A) Jade1 KO1 mIMCD3 cells were transiently transfected with either Flag-tagged Jade1L, Jade1S, or a control plasmid. Pulldown was performed with anti-Flag (M2) sepharose beads. Heatmap of average iBAQ values for proteins which were never measured in the control samples, but in either all replicates for Jade1L, all replicated for Jade1S, or all replicates of both. The protein with the highest iBAQ is Jade1 for both, the Jade1S and the Jade1L pulldown. (B/C) Quantitative analysis of the Jade1L (B) \& Jade1S (C) interactome. Proteins on the right side, above the $p=0.05$ line, are determined significantly enriched. Proteasomal components are highlighted in magenta, known JADE1/Jade1 interactors based on known interactors annotated in BioGrid (54) in green.

\section{Supplementary Figure Legends}

Supplemental Figure S1: Sequence alignment and expression profiles of the JADE protein family members. (A) Protein identity matrix for human and mouse JADE family paralogues. Protein sequences were obtained from Uniprot (55) and the identity score was calculated using Clustal Omega (56). (B) Protein Expression Levels for JADE1 across different tissues. Based on tissue micro arrays obtained from The Human Protein Atlas version 19.3 and Ensembl version 92.38. Proteomic data available from https://v19.proteinatlas.org/download/normal_tissue.tsv.zip . (57). (C) RNA expression profiles across 
different tissues for $J A D E 1 / 2 / 3$. Consensus transcript expression levels based on transcriptomic data from HPA, GTEx and FANTOM5. Normalized expression (NX) based on the maximum NX for each gene in the different data sets. Data is obtained from The Human Protein Atlas v 19.3 and Ensembl version
92.38.
Transcriptomic
data
available
from https://v19.proteinatlas.org/download/rna_tissue_consensus.tsv.zip. (57). (D) Gene expression data of single cell transcriptomics of mouse kidney. Shown is the percentage of cells expressing each Jade gene in the different cell clusters: endothelial, vascular, and descending loop of Henle (Endo), podocyte (Podo), proximal tubule (PT), ascending loop of Henle (LOH), distal convoluted tubule (DCT), collecting duct principal cell (CD-PC), collecting duct intercalated cell (CD-IC), collecting duct transitional cell (CDTrans), fibroblast (Fib), macrophage (Macro), neutrophil (Neutro), lymphocyte (lymph), natural killer cell (NK)(26).

Supplemental figure S2: Generation and validation of Jade1/2/3 mIMCD3 mutant cell lines. (A) Selection of sgRNAs targeting exons containing the first and the second PHD domain. sgRNA selection was based on location and off-target scores using Wellcome Sanger Institute Genome Editing (https://wge.stemcell.sanger.ac.uk/) (40). (B) Sanger sequencing of mRNA confirms mutations.

Supplemental figure S3: Protein turnover machinery is upregulated upon loss of Jade family members. (A-C) Comparison of the two different mutant cell lines for Jade1 (A), Jade2 (B), and Jade3 (C). The fold change of KO1 is depicted on the $\mathrm{x}$-axis, the fold change of the $\mathrm{KO} 2$ on the $\mathrm{y}$-axis. Proteins are highlighted according to their significance as indicated in the figure. (D) Scatter plot with the t-test differences in protein expression of the Jade1 KO2 mutant cells vs the wildtype cells on the $\mathrm{x}$-axis and the statistical significance (- $\log _{10}$ Student's t-test $p$-value) on the $y$-axis. Ribosomal proteins are highlighted in magenta, tRNA ligases in blue, and elongation factors in green. (E, F) Heatmap of the proteins highlighted in (D) based on logarithmic LFQ values for all seven cell lines to visualize the upregulation of ribosomal proteins (E), tRNA ligases, and elongation factors (F) across Jade-deficient cell lines.

\section{Supplemental figure S4: Transcriptomic analysis reveals no major regulation of the proteasome. (A)} PCA plot of the gene expression data of WT, Jade1 KO1 and KO2 cell lines. Depicted are the first two principal components (PC1 and PC2). The axes represent the percentages of variation explained by the principal components. The replicates of the different groups cluster together and the difference between mutant and wildtype groups explains the largest variance. PCA plots of Jade2 (B) and Jade3 (C) cell lines show clustering of the groups. Here, the second component accounts for the difference between mutant and wildtype cell lines. (D-F) Comparison of the two different mutant cell lines for Jade1 (D), Jade2 (E), and Jade3 (F). The fold change of KO1 is depicted on the $\mathrm{x}$-axis, the fold change of the $\mathrm{KO} 2$ on the $\mathrm{y}$-axis. Genes are highlighted according to their significance as indicated in the figure. 
Supplemental figure S5: Transcriptomic analysis reveals no major regulation of the proteasome. (A) Venn diagram depicting the overlap between significantly regulated genes across the Jade-deficient cell lines. The Jade1 section includes all proteins that are significantly regulated ( $p$-value $<0.05$ ) in either one or both of the Jade1-deficient cell lines. The Jade2 and Jade3 sections generated in the same way. (B) Scatter plot with the log2FoldChange in gene expression of the Jade1 KO2 mutant cells vs the wildtype cells on the $x$-axis and the statistical significance (-log adj. p-value) on the y-axis. Genes of proteasomal components are highlighted in magenta. (C) Heatmap of the genes highlighted in $(H)$ based on logarithmic normalized counts for all seven cell lines to visualize that the proteasomal components are not upregulated on a transcriptional level across Jade-deficient cell lines. (D-F) Heatmap of ribosomal genes (D), genes encoding for tRNA-ligases (E), and genes encoding for transcription initiation and elongation factors (F). Logarithmic normalized counts for all seven cell lines are shown.

\section{Supplemental tables}




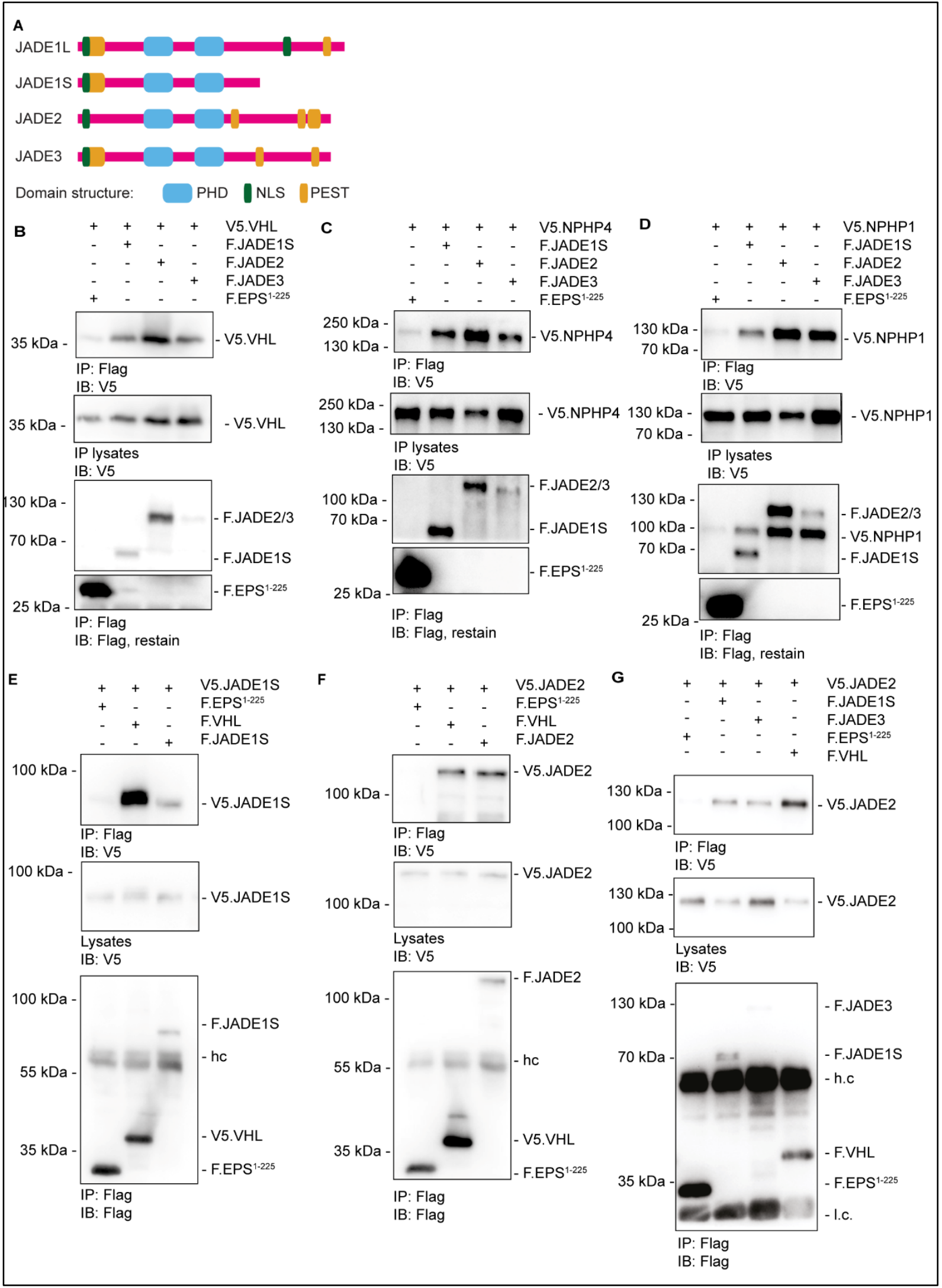

Figure 1: All JADE protein family members co-precipitate with the ciliopathy proteins NPHP1, NPHP4, and pVHL. (A) Domain structure of the human JADE proteins. PHD - Cys4HisCys3 plant homeo domain; NLS - nuclear localization signal; PEST - protein degradation amino acid sequence. Domain information 
-X - (K/R)). (B-D) HEK293T cells were transiently transfected with (B) V5-tagged pVHL (V5.VHL), (C)

794 V5-tagged Nphp4 (V5.NPHP4), and (D) V5-tagged Nphp1 (V5.NHPH1), and either FLAG-tagged JADE1S

795 (F.JADE1S), Jade2 (F.JADE2), Jade3 (F.JADE3) or a control protein (F.EPS ${ }^{1-225}$ ). Immunoprecipitates with

796 an anti-FLAG antibody were analyzed by immunoblotting with antibodies (anti-V5; anti-FLAG) as indicated. V5.VHL, V5.NPHP1, and V5.NPHP4 all coimmunoprecipitate with F.Jade1S/2/3 but not with F.EPS ${ }^{1-225}$. Blots are representative for at least three independent experiments. (E,F) HEK293T cells were transiently co-transfected with (E) V5.JADE1S and FLAG.JADE1S or (F) V5.JADE2 and FLAG.JADE2.

800 FLAG.pVHL and FLAG.EPS ${ }^{1-225}$ served as positive and negative control, respectively.

801 Immunoprecipitates with an anti-FLAG antibody were analyzed by immunoblotting with antibodies as

802 indicated. V5.JADE1S and V5.JADE2 both co-immunoprecipitate with the respective FLAG-tagged JADE 803 protein. (G) HEK293T cells were transiently transfected with V5.JADE2 and either FLAG-tagged JADE1S, 804 JADE3, pVHL, or EPS ${ }^{1-225}$. Immunoprecipitates with an anti-FLAG antibody were analyzed by 805 immunoblotting with antibodies (anti-V5; anti-FLAG) as indicated. V5.JADE2 coimmunoprecipitate 806 with F.JADE1S, F.JADE3, and F.VHL but not with F.EPS ${ }^{1-225}$ (h.c.: heavy chain, I.c.: light chain). 


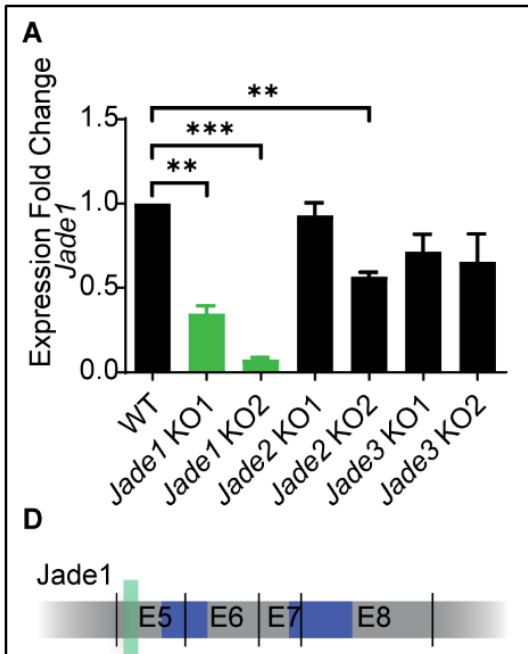

Peptide A

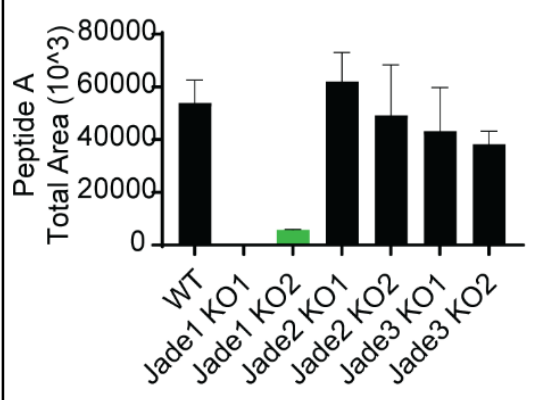

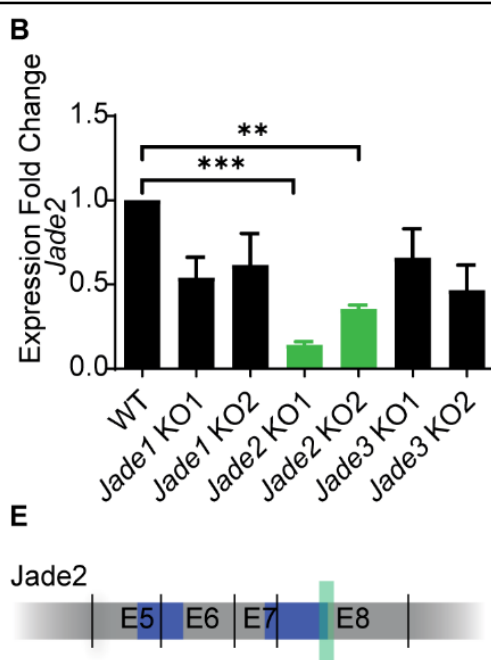

Peptide B
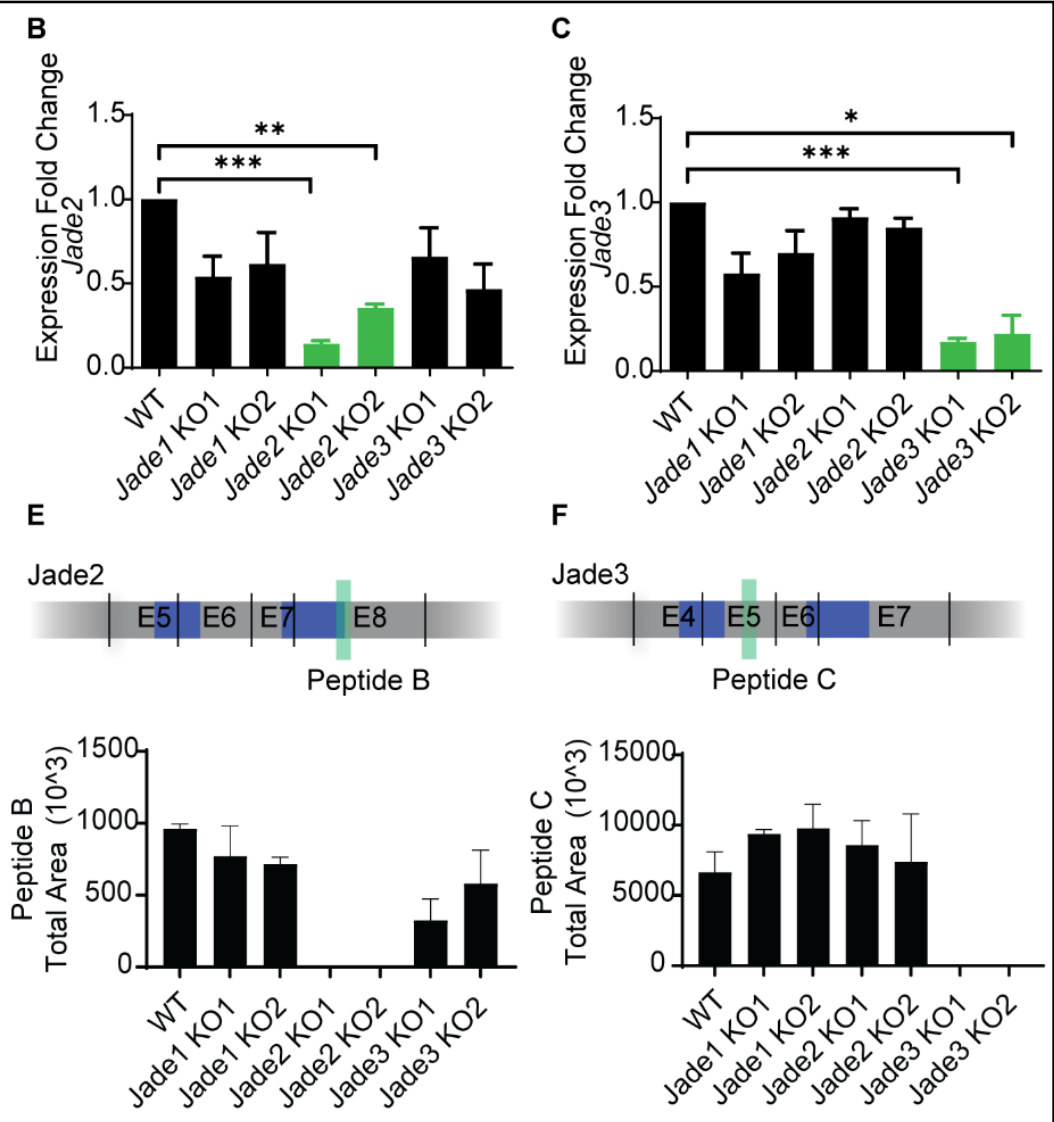

Figure 2: Deletion of one specific Jade protein does affect neither transcription nor expression of the

remaining family members. (A-C) qPCR data comparing the expression fold change of each targeted gene across the different cell lines. Only the expression of the targeted gene in the respective mutant cell lines is significantly reduced, while the other Jade mRNAs are not significantly altered. $(\mathrm{N}=3$, students t-test, $* p \leq 0.05, * * p \leq 0.01, * * * p \leq 0.001$ ) (D-F) Parallel reaction monitoring (PRM) assay for (D) Jade1, (E) Jade2, and (F) Jade3 comparing the peak areas for the selected peptides across mIMCD3 WT and Jade mutant cell lines for protein quantification. The localization of peptides selected for PRM assay is depicted above the bar charts. $(\mathrm{N}=3)$ 

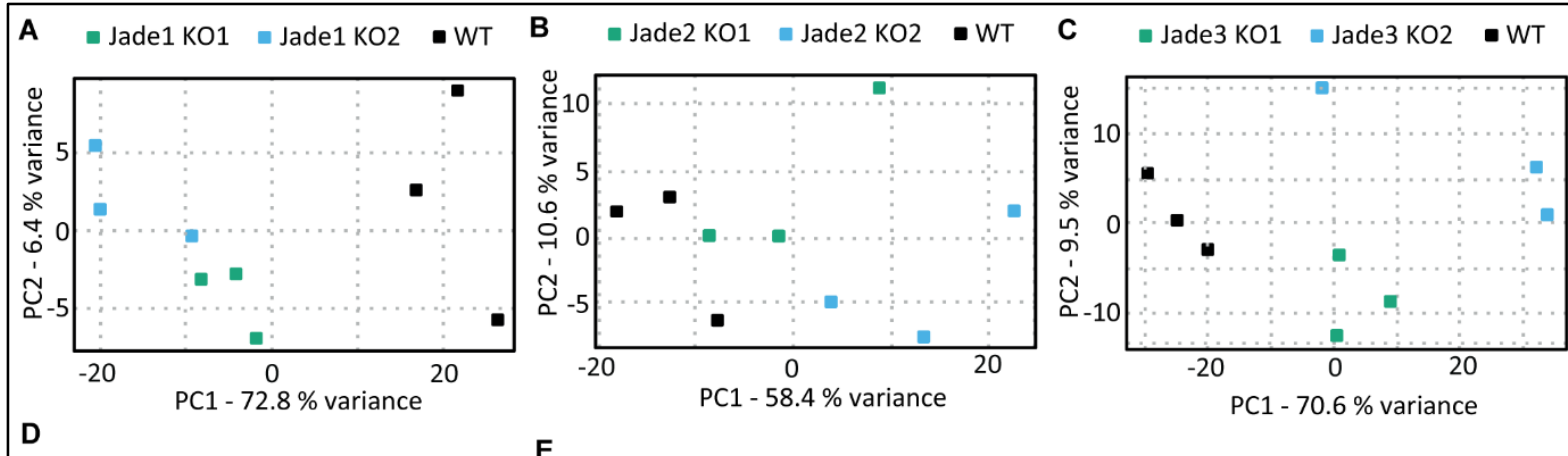

D

E
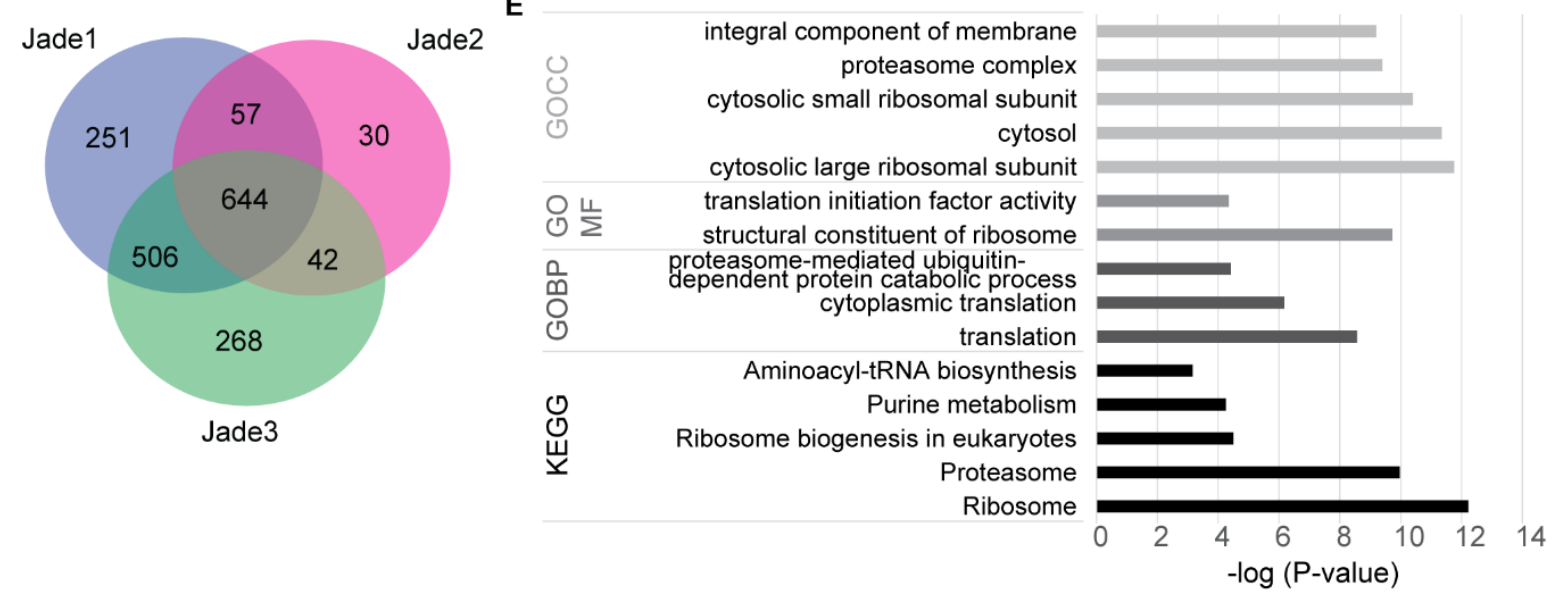

Ubiquitin proteasome system

G
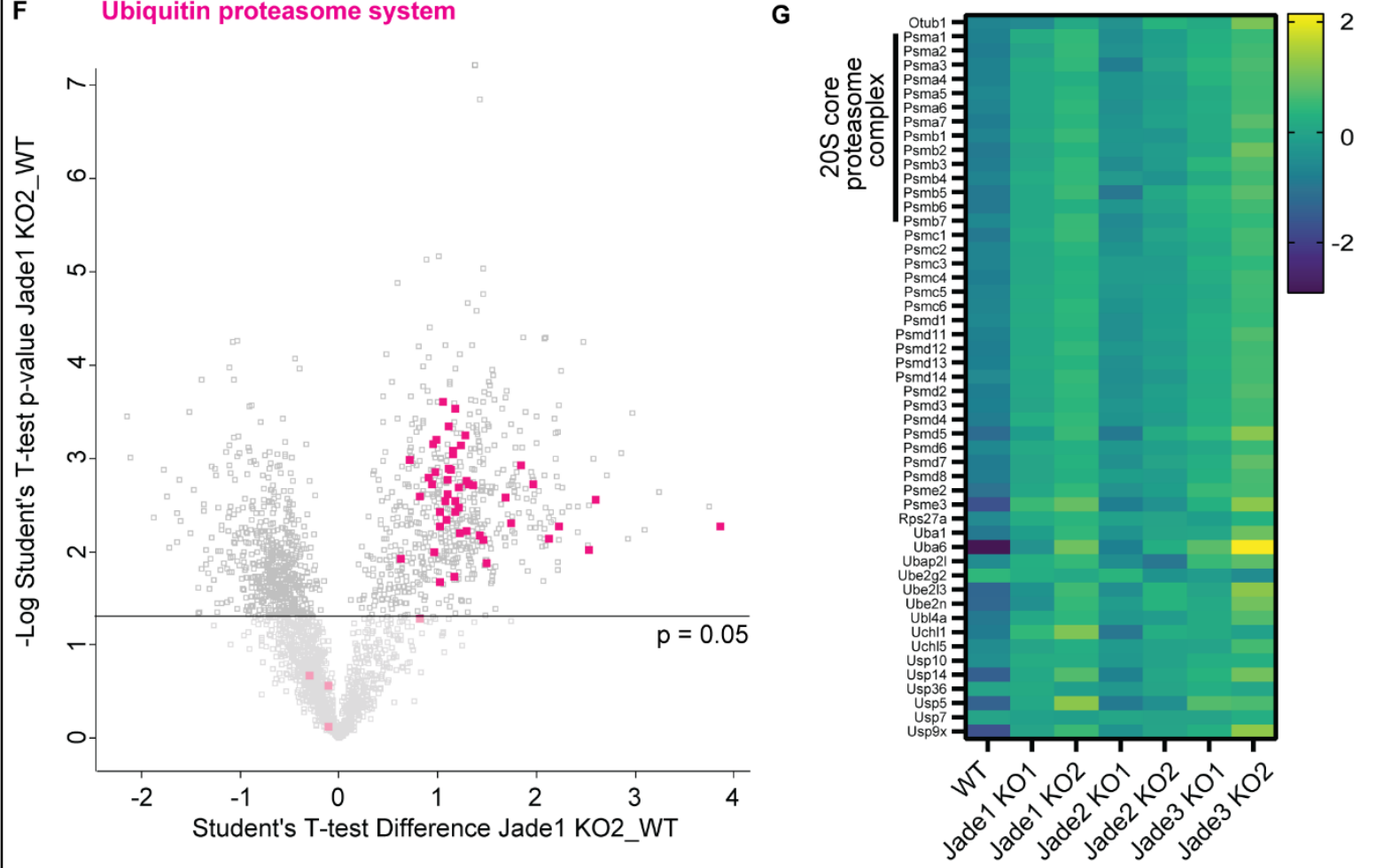

Figure 3: Proteomic analysis reveals upregulation of the proteasome in cell lines lacking either one

of the Jade family members. (A-C) Principal component analysis (PCA) plot of the protein expression data of WT, Jade1 (A), Jade2 (B), and Jade3 (C) KO1 and KO2 cell lines. Depicted are the first two principal components (PC1 and PC2). The axes represent the percentages of variation explained by the principal components. The replicates of the different groups cluster together and the difference between mutant and wildtype groups explains the largest variance. (D) Venn diagram depicting the 
824 overlap between significantly regulated proteins across the Jade-deficient cell lines. The Jade1 section

825 includes all proteins that are significantly regulated $(p$-value $<0.05)$ in either one or both of the Jade1-

826 deficient cell lines. The Jade2 and Jade3 sections generated in the same way. (E) GO and KEGG pathway

827 annotation based on a Fisher exact test of the 644 proteins found to be regulated in all Jade mutant

828 cell lines. (F) Representative scatter plot with the t-test differences in protein expression of the Jade1

$829 \mathrm{KO} 2$ mutant cells vs the wildtype cells on the $\mathrm{x}$-axis and the statistical significance $\left(-\log _{10}\right.$ Student's t-

830 test $p$-value) on the $y$-axis. Proteins associated with the ubiquitin proteasome system are highlighted

831 in magenta. (G) Heatmap of the proteins highlighted in (F) based on logarithmized LFQ values for all

832 Jade-deficient cell lines and the control line visualizing the upregulation of proteasome-associated

833 proteins.

834 
A

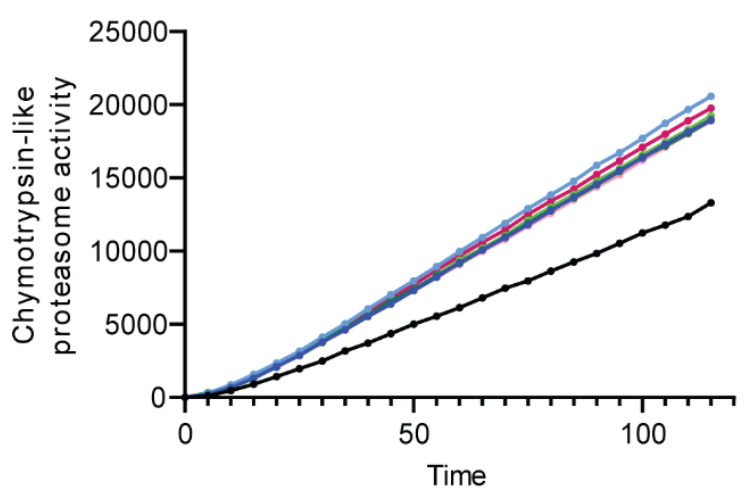

$\rightarrow$ WT

$\rightarrow$ Jade1 KO1

$\rightarrow$ Jade1 KO2

$\rightarrow$ Jade2 KO1

$\rightarrow$ Jade2 KO2

$\rightarrow$ Jade3 KO1

$\rightarrow$ Jade3 KO2

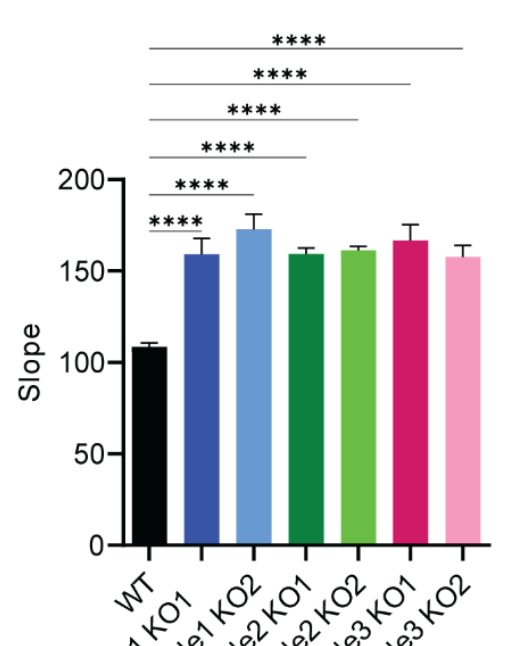

B

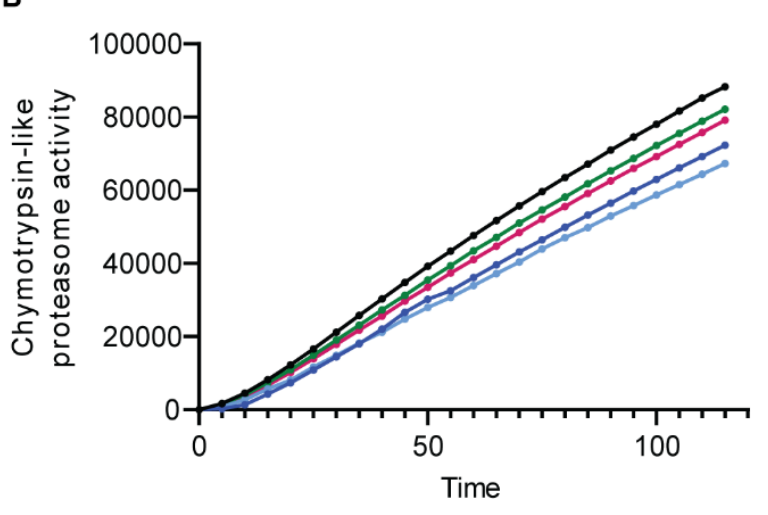

$$
\begin{aligned}
& \rightarrow \text { Control } \\
& \rightarrow \text { Jade1S } \\
& \rightarrow \text { Jade1L } \\
& \rightarrow \text { Jade2 } \\
& \rightarrow \text { Jade3 }
\end{aligned}
$$

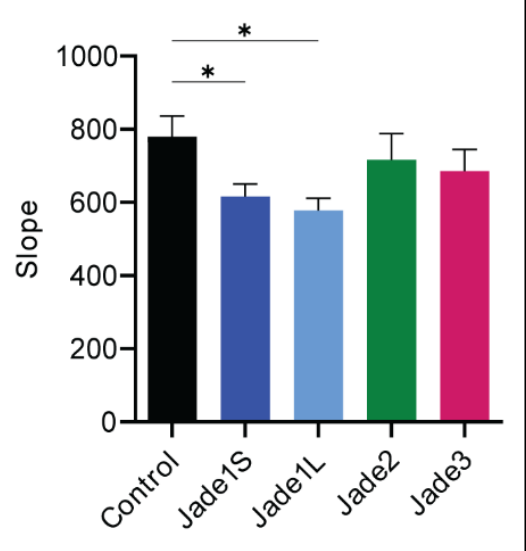

Figure 4: Increased proteasome activity observed in Jade-deficient cell lines. (A) Measurement of the proteasome activity in wildtype and Jade-deficient mIMCD3 cell lines. The proteasome activity of Jade1, Jade2, or Jade3-deficient cell lines is increased compared to the wildtype control. Quantification of the slope as readout for proteasome activity ( $N=3$, one-way ANOVA with Dunnett's post-hoc test, ${ }^{* * *} p \leq 0.0001$ ) (B) Measurement of the proteasome activity in HEK293T cell with either Jade1S, Jade1L, Jade2, Jade3, or Podocin (control) overexpressed. All cell lines with overexpressed Jade proteins show a decreased activity compared to the control with only Jade1S/L reaching statistical significance. Quantification of the slope as readout for proteasome activity ( $\mathrm{N}=3$, one-way ANOVA Dunnett's post-hoc test, $* p \leq 0.05$ ) 


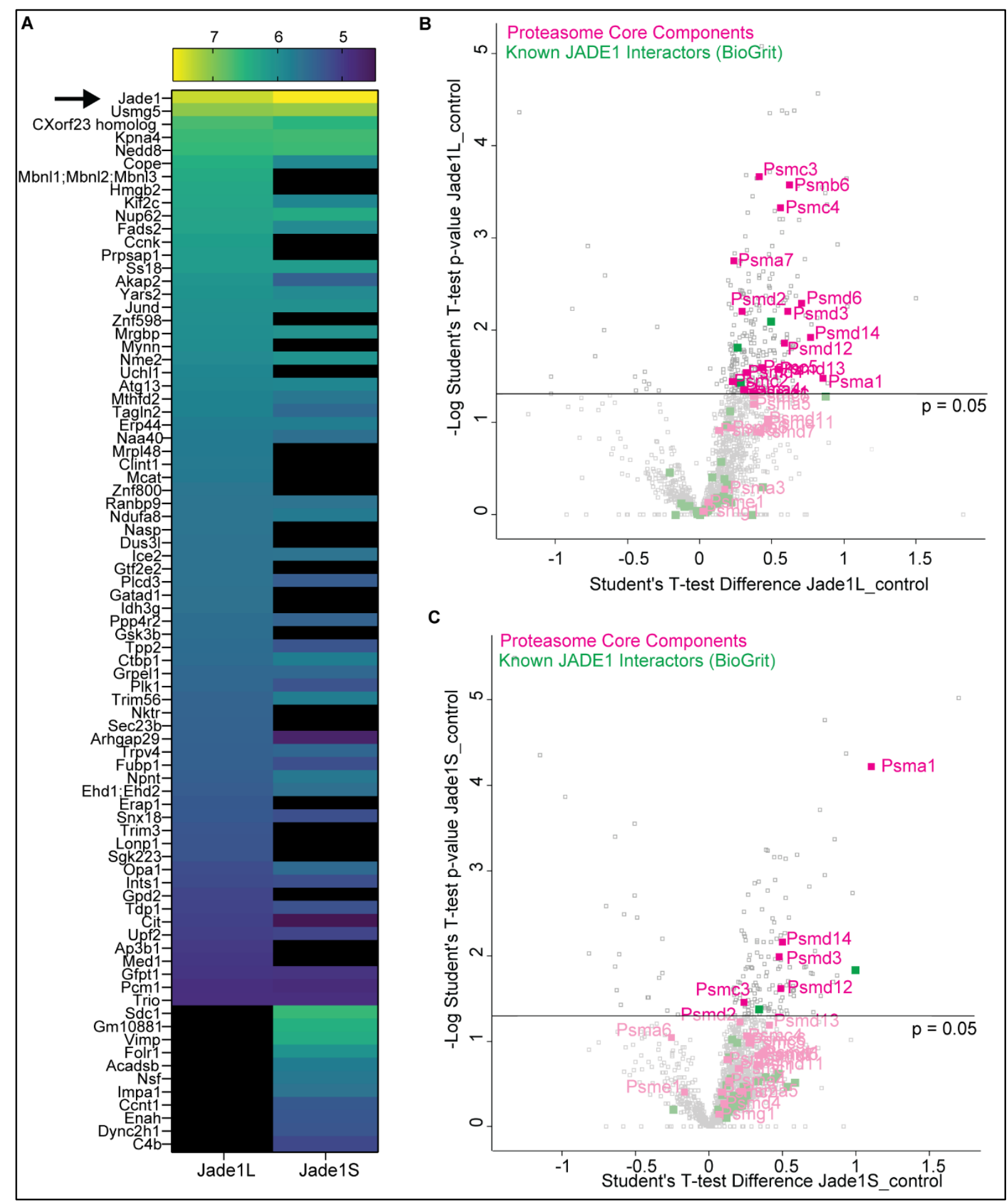

Figure 5: Interactome analysis reveals a link between Jade1L/S proteins and proteasomal

components. (A) Jade1 KO1 mIMCD3 cells were transiently transfected with either Flag-tagged Jade1L, Jade1S, or a control plasmid. Pulldown was performed with anti-Flag (M2) sepharose beads. Heatmap of average $\mathrm{iBAQ}$ values for proteins which were never measured in the control samples, but in either all replicates for Jade1L, all replicated for Jade1S, or all replicates of both. The protein with the highest iBAQ is Jade 1 for both, the Jade1S and the Jade1L pulldown. (B/C) Quantitative analysis of the Jade1L (B) \& Jade1S (C) interactome. Proteins on the right side, above the $p=0.05$ line, are determined 
bioRxiv preprint doi: https://doi.org/10.1101/2021.10.01.462752; this version posted October 1, 2021. The copyright holder for this preprint (which was not certified by peer review) is the author/funder, who has granted bioRxiv a license to display the preprint in perpetuity. It is made available under aCC-BY-NC-ND 4.0 International license.

853 significantly enriched. Proteasomal components are highlighted in magenta, known JADE1/Jade1

854 interactors based on known interactors annotated in BioGrid (54) in green. 